\title{
Editorial
}

\section{Image Enlargement Using Multiple Sensors}

\author{
Wei Wu, ${ }^{1}$ Marco Anisetti, ${ }^{2}$ Chehri Abdellah, ${ }^{3}$ and Gwanggil Jeon ${ }^{4}$ \\ ${ }^{1}$ College of Electronics and Information Engineering, Sichuan University, Chengdu 610064, China \\ ${ }^{2}$ Dipartimento di Informatica, Università degli Studi di Milano, Via Bramante 65, 26013 Crema, Italy \\ ${ }^{3}$ School of Electrical Engineering and Computer Science, University of Ottawa, 800 King Edward Avenue, Ottawa, \\ ON, Canada K1N 6N5 \\ ${ }^{4}$ Department of Embedded Systems Engineering, College of Information and Technology, Incheon National University, \\ 119 Academy-ro, Yeonsu-gu, Incheon 22012, Republic of Korea
}

Correspondence should be addressed to Gwanggil Jeon; gjeon@inu.ac.kr

Received 10 September 2015; Accepted 13 September 2015

Copyright (C) 2016 Wei Wu et al. This is an open access article distributed under the Creative Commons Attribution License, which permits unrestricted use, distribution, and reproduction in any medium, provided the original work is properly cited.

Image sensing is generally performed with multiple spectral sensors. For example, combination of three sensors (red, green, and blue) is used for color image reproduction, and electrooptical and infrared sensors are used for surveillance and satellite imaging, respectively. The resolution of each sensor can be intensified by taking the other sensors into account and applying correlations between different sensors. There are various successful applications of image enlargement using multiple sensors and even multimodal sensors. However, there still are several open issues in sensor processing which can be explained by signal processing-based image enlargement using redundancy among the sensors.

The articles contained in the present issue include both reviews and basic scientific studies focused on image enlargement using multiple spectral sensors to improve image quality. This issue comprises the description of image enlargement methodologies which uses embedded system, signal processing (audio and feature extraction), denoising, regularization for error minimizing, super resolution, and artificial enhancement such as neural network.

Pulse coupled neural network (PCNN) has been widely used in image processing. The 3D binary map series (BMS) generated by PCNN can be treated as the basis of extracting 1D oscillation time series (OTS) for an image, since they effectively describe image features such as edges and regional distribution.

However, the traditional methods using BMS require improvements for being effectively applied in some specific field of image processing such as face recognition. The contribution by R. Nie et al. "Facial Feature Extraction Using Frequency Map Series in PCNN" computed 2D entropy for every map in frequency map series (FMS) obtained from BMS, and then 3D FMS was transformed into 1D OTS (OTSFMS). The authors proved that OTS-FMS feature has good geometry invariance for the facial image and contains the space structure information of the image.

The face shape model is used together with the scope of detection and recognition for many applications; however, there are still some imitations in the current state of the art solutions especially for the aspects related to feature detection and tracking. The contribution by S. Kang and S. Kim "Patch Classifier of Face Shape Outline Using Gray-Value Variance with Bilinear Interpolation" proposed a method to classify whether the outline in a face shape model is properly fitted to feature points. The authors implemented a classifier using this characteristic and achieved good accuracy through the patch classifier.

Image is a $2 \mathrm{D}$ signal; thus any type of signal processing approaches even audio processing techniques can be used as inspiration for developing image processing. The contribution by S. Ryu et al. "Active Suppression of Narrowband Noise by Multiple Secondary Sources" extensively investigated in both theory and real-time control experiment on the active suppression of narrowband noise. After analyzing the primary and secondary paths of the duct system, an acoustic narrowband signal was chosen as a primary noise and 
the impulse responses were implemented as the secondary path models. The authors proved that the lower quality factor cancels the more primary noise as defined in the theory although the attenuation levels are not exactly and inversely proportional to the quality factor.

Image processing is nowadays applied in many application fields including automotive. Camera-based advanced driver assistance systems (ADAS) have been introduced to assist the drivers and ensure their safety under various driving conditions like in the case of low visibility and lower contrast due to foggy conditions. The contribution by F. Hussain and J. Jeong "Visibility Enhancement of Scene Images Degraded by Foggy Weather Conditions with Deep Neural Networks" presented a new approach to provide a solution to this problem by employing deep neural networks and mathematically modelling fog in an image. This paper achieved two advantages, "real-time operation" and "robustness/generalization for various unseen image data."

Image enlargement method as the one specific for ADAS system is in general simultaneously applied with image denoising. Image denoising methods are often based on the minimization of an appropriately defined energy function. The contribution by L. Guo et al. "An Edge-Preserved Image Denoising Algorithm Based on Local Adaptive Regularization" adaptively adjusted denoising degree of noisy image by adding spatial variable fidelity term.

The spectrogram image analysis of error signals can be used in the active noise suppression. The contribution by J. Kim et al. "Spectrogram Image Analysis of Error Signals for Minimizing Impulse Noise" presented the theoretical and experimental study on the spectrogram image analysis of error signals for minimizing the impulse input noises in the active suppression of noise. The authors proved that the suggested algorithm worked with a nice stability and performance against impulse noises, applying it for practical active noise control systems.

There have been many image enlargement methods, which are based on sparse coding especially in single image superresolution reconstruction. When applied to infrared (IR) images, the traditional sparse representation-based (SR) image reconstruction methods suffer from (i) lack of detailed information, (ii) fixed size patch-based dictionary, (iii) universal and overcomplete dictionary. The contribution by X. Yang et al. "Multiscale and Multitopic Sparse Representation for Multisensor Infrared Image Superresolution" overcame the above issues by combining the information from multisensors to improve the resolution of the IR image. In addition, they used multiscale patches to represent the image in a more efficient manner. Finally they partition the natural images into documents. Then they group such documents to determine the inherent topics using PLSA and to learn the sparse dictionary of each topic.

The Embedded Graphics Processing Unit (EGPU) has assumed more processing tasks within embedded devices, requiring high computational capacity. The contribution by Y. Wang et al. "A Tile-Based EGPU with a Fused Universal Processing Engine and Graphics Coprocessor Cluster" proposed general-purpose computing and $3 \mathrm{D}$ graphics rendering. The authors demonstrated that the proposed EGPU can be used in a System on Chip (SoC) configuration connected to sensors with the scope of accelerating its processing and creating a proper balance between performance and cost.

Stitching is one of efficient methods for images enlargement by stitching adjacent images which contain overlapping regions even though they are obtained through separate image sensors. The contribution by K. Jun and S. Kim "Feature Coverage Indexes for Dual Homography Estimation in Constructing Panorama Image" proposed dual homography method. The authors presented three feature coverage indexes which evaluate the stitching performance of feature detectors and predict the outcomes of the stitching.

The theoretical resolution of $35 \mathrm{~mm}$ digital cinema system is greater than that of $2 \mathrm{~K}(2048 \times 1080)$ digital cinema. Today's digital cinema system equips hundreds of $4 \mathrm{D}$ chairs and the environmental directors; it is nearly infeasible for the legacy system to control. The contribution by E. Kim et al. "RS485 Image Sensor for Digital Cinema System" designed and implemented a new system, which makes hundreds of 4D chairs and the environmental directors be controlled simultaneously by exploiting RS485 network topology and its repeaters.

Digital image compression plays an extremely important role in the transmission and storage of digital image data. Image compression is the process of effectively coding digital images to reduce the number of bits required to represent an image. The contribution by F. Hussain and J. Jeong "Efficient Deep Neural Network for Digital Image Compression Employing Rectified Linear Neurons" proposed a compression technique for still digital images which uses deep neural networks (DNNs) and rectified linear units (ReLUs). The authors proved that ReLUs well establish an efficient gradient propagation and the proposed system is efficient in terms of computations making these networks suitable for real-time compression systems.

Due to the rapid advancement of the airborne sensors and spaceborne sensors, large volumes of fully polarimetric synthetic aperture radar (PolSAR) data are available, but they are too complex to interpret difficultly. The contribution by S. Zhang et al. "Modified Hybrid Freeman/Eigenvalue Decomposition for Polarimetric SAR Data" proposed modified hybrid Freeman/eigenvalue decomposition method for the coherency matrix derived from the fully PolSAR sensors. The proposed method used a real unitary transformation on the coherency matrix to release correlations between the copolarized term and cross polarized term.

Hyperspectral image obtains terrestrial information in various contiguous and narrow spectral bands, and it is attracting more and more researchers due to its wide applications in aerial and space imagery fields. The contribution by J. Wang et al. "Superresolution of Hyperspectral Image Using Advanced Nonlocal Means Filter and Iterative Back Projection" introduced an efficient superresolution algorithm based on advanced nonlocal means (NLM) filter and iterative back projection for hyperspectral image. The authors proved that their method is able to recover the high-resolution image by iteratively minimizing the reconstruction error from the given low-resolution image which is blurred due to the noise. 
We hope that this special issue would shed light on major developments in the area of image enlargement using multiple sensors and attract attention by the scientific community to pursue further investigations leading to the rapid implementation of these sensor technologies.

\section{Acknowledgments}

We would like to express our appreciation to all the authors for their informative contributions and the reviewers for their support and constructive critiques in making this special issue possible. This work was supported under the framework of international cooperation program managed by National Research Foundation of Korea (no. 2014000756) and by National Natural Science Foundation of China (no. 61271330 and no. 6141101009).

Wei Wu

Marco Anisetti

Chehri Abdellah

Gwanggil Jeon 


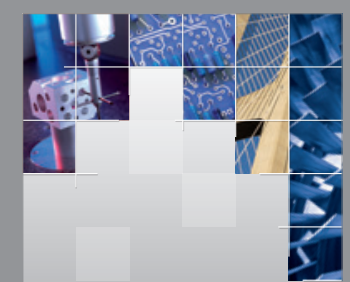

\section{Enfincering}
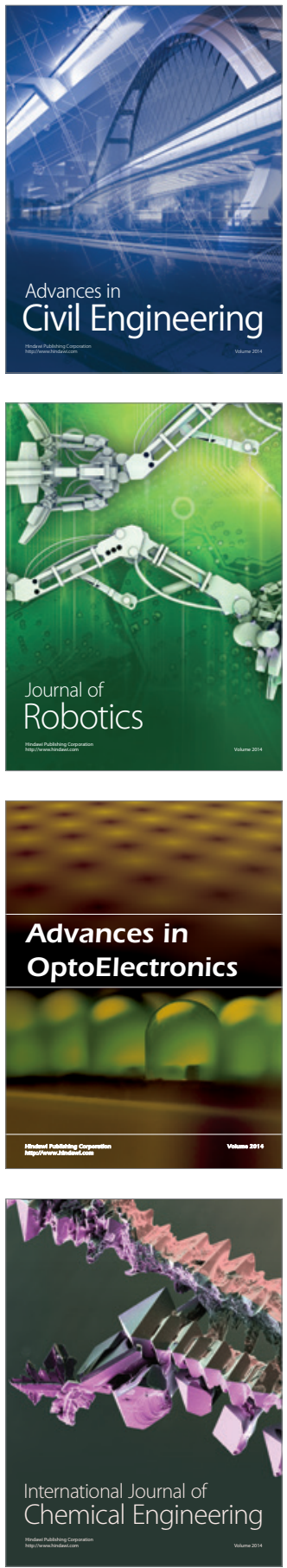

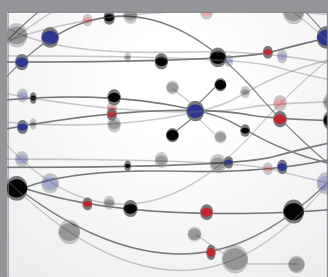

The Scientific World Journal

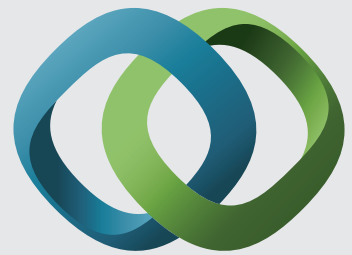

\section{Hindawi}

Submit your manuscripts at

http://www.hindawi.com
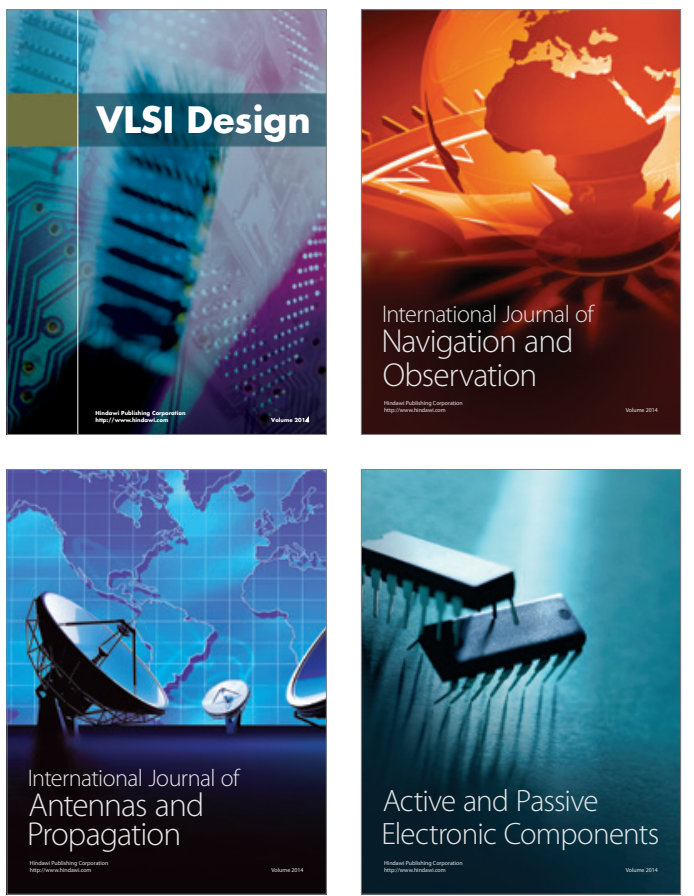
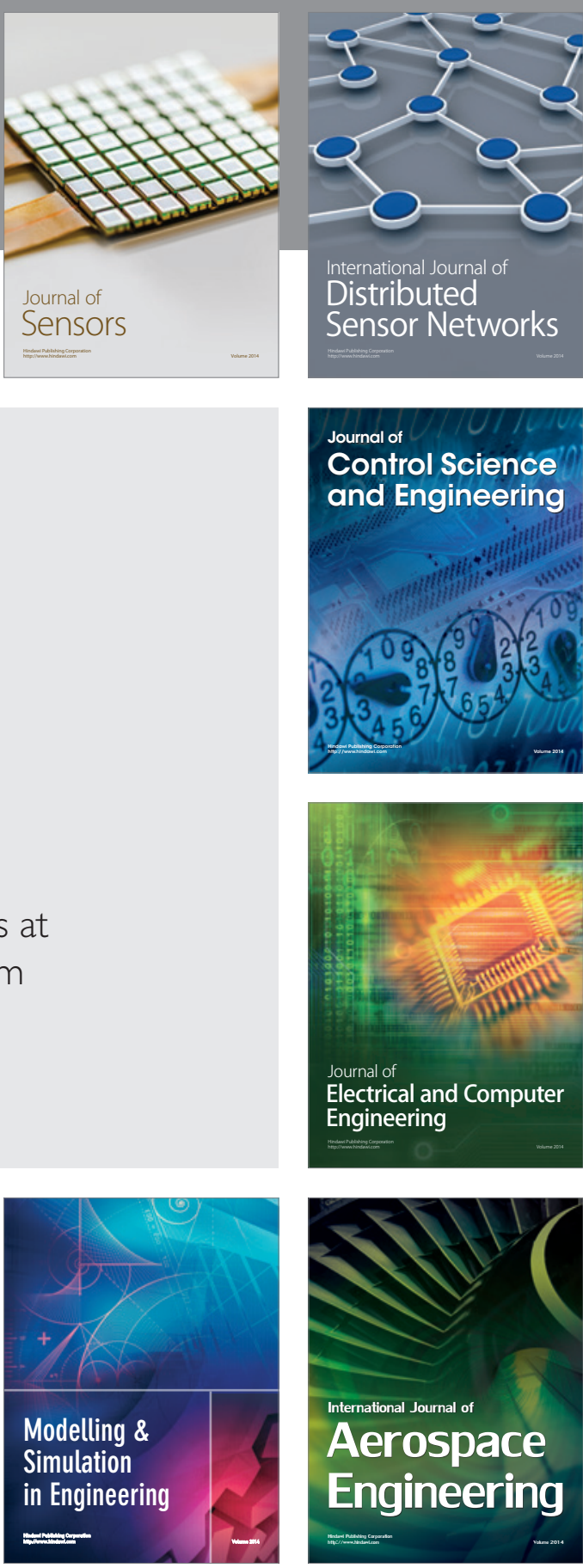

International Journal of

Distributed

Sensor Networks

Journal of

Control Science

and Engineering
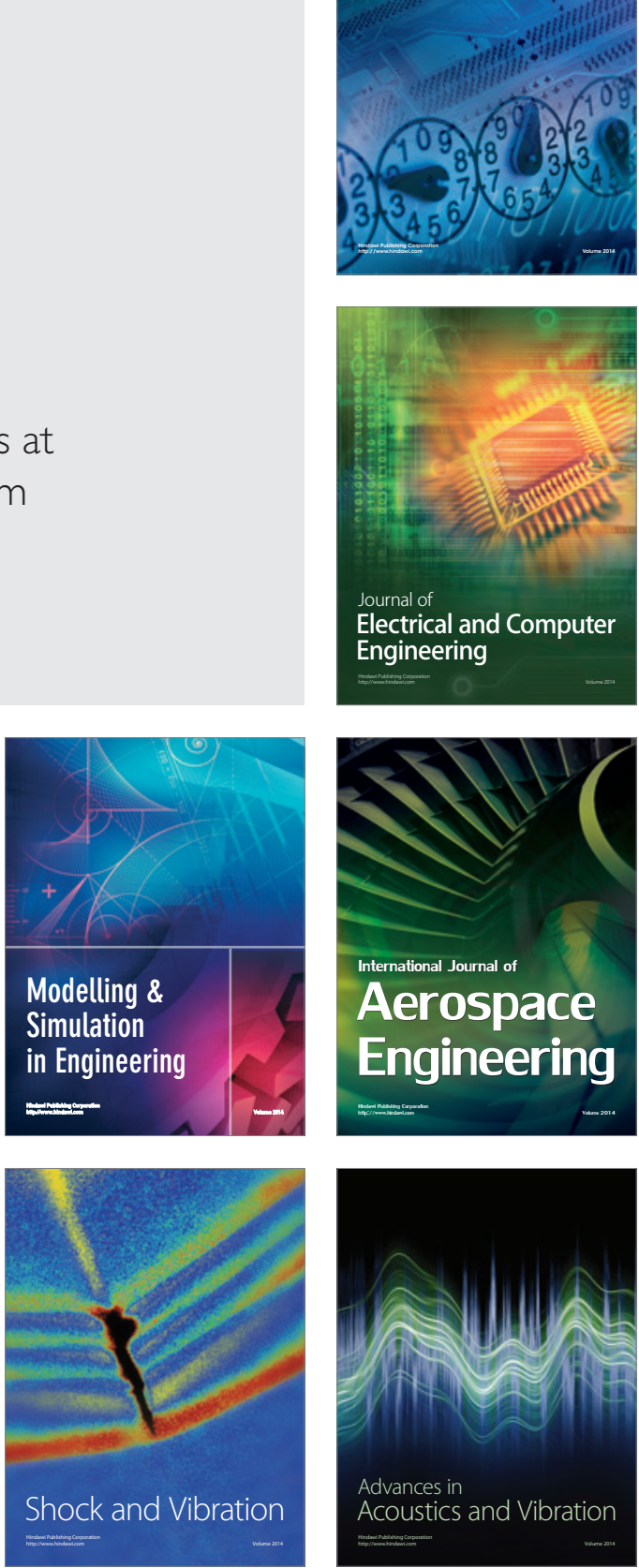